\title{
Cumplimiento de la Guía de Práctica Clínica de EPOC por médicos de primer nivel
}

\author{
Eloisa González-González,* $\bowtie$ Margarita Fernández-Vega, \\ Consuelo González-Salinas, *, Rafael de Jesús Hernández-Zenteno*
}

\author{
*Centro de Salud, Secretaría de Salud; \#nstituto Nacional de Enfermedades Respiratorias Ismael Cosío Villegas, Ciudad de México; \\ \$Universidad Nacional Autónoma de México. \\ Trabajo recibido: 19-I-2016; aceptado: 01-III-2016
}

\begin{abstract}
RESUMEN. La enfermedad pulmonar obstructiva crónica (EPOC) es el problema respiratorio de mayor relevancia e impacto socioeconómico en el mundo. A pesar de ser prevenible y contar con métodos de diagnóstico oportuno, hay evidencia de que es una enfermedad subdiagnosticada y subtratada. Objetivo: Determinar el cumplimiento de las recomendaciones de las Guías de Práctica Clínica de la EPOC por el personal médico de un centro de salud. Material y métodos: Estudio descriptivo y comparativo, transversal y ambispectivo. Se analizaron todos los expedientes de pacientes con EPOC confirmado entre 2014-2015, y se contrastó la historia clínica y notas médicas con las recomendaciones de la Guía de Práctica Clínica (GPC). Resultados: Se analizaron 32 notas médicas; de las cuales los médicos residentes cumplieron las recomendaciones en un $49.6 \%$, los médicos familiares adscritos $33.2 \%$ y los médicos generales adscritos $31.6 \%$. Conclusión: Existe un bajo apego a la GPC. El bajo cumplimiento se observó en la mayoría de las recomendaciones de la GPC. Se demostraron diferentes porcentajes de cumplimiento con base en el perfil del médico de primer contacto.
\end{abstract}

Palabras clave: Guías, EPOC, atención primaria.

\begin{abstract}
Chronic obstructive pulmonary disease (COPD) is the most relevant cause of respiratory problems with a high social-economic impact in the world. Despite being a preventable disease and have an early diagnosis method, there is evidence of underdiagnosis and undertreatment. Objective: To determine the adherence to the recommendations of a COPD Clinical Practice Guidelines (CPG) by primary care physicians at a primary care clinic. Material and methods: Descriptive and comparative, cross-sectional and ambispective study, of medical records of patients with confirmed COPD from 2014 to 2015. Each medical history and medical note was compared with the CPG recommendations; for the statistical analysis, descriptive and comparative methods were obtained for each recommendation. Results: 32 medical notes were analyzed; family medicine residents met $49.6 \%$ of the recommendations; staff family medicine specialist followed $33.2 \%$ of the recommendations and staff general practitioners $31.6 \%$. Conclusion: There is a low adherence to the GPC. This low adherence was observed at most of the recommendations. It was demostrated different percentages of adherence according to physician profile.
\end{abstract}

Key words: Guidelines, COPD, primary care.

\section{INTRODUCCIÓN}

La enfermedad pulmonar obstructiva crónica (EPOC) es una enfermedad inflamatoria, progresiva, prevenible y tratable, caracterizada por obstrucción persistente del flujo aéreo espiratorio., ${ }^{1,2}$ Se considera una condición de gran relevancia con alto impacto socioeconómico en el mundo, y una morbimortalidad en incremento sostenido ${ }^{3}$ que lo hacen un problema prioritario de salud pública. ${ }^{4,5}$

En México, su prevalencia es de $7.8 \%$ y tiene un subdiagnóstico de casi $90 \% .^{3}$ En el Instituto Nacional de Enfermedades Respiratorias Ismael Cosío Villegas (INER), ocupó el tercer lugar en visitas a urgencias y el segundo lugar en la consulta externa en $2011 .{ }^{6}$ Para el 2020 será la tercera causa de muerte en el mundo ${ }^{4,7}$ y la quinta causa en años de vida perdidos ajustados por invalidez. ${ }^{8,9}$ Además, la EPOC genera un elevado

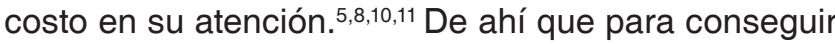
un diagnóstico temprano y un abordaje oportuno que disminuya el impacto de la enfermedad existen diversas guías para la prevención, diagnóstico y tratamiento. ${ }^{8}$ En nuestro país, resalta la Guía de Práctica Clínica (GPC) para (EPOC) ${ }^{8}$ que incorpora evidencias y recomendaciones de intervenciones de prevención primaria, cribado y tratamientos. ${ }^{8,12}$ Mediante encuestas a médicos de primer contacto se ha demostrado un bajo 
conocimiento y pobre uso de las guías de EPOC..$^{13}$ Este apego limitado no ha sido evaluado mediante la revisión de expedientes clínicos.

El objetivo del presente estudio fue determinar el cumplimiento de la GPC para la prevención, diagnóstico, tratamiento y referencia de pacientes con EPOC por médicos de un centro de salud.

\section{MATERIAL Y MÉTODOS}

Estudio descriptivo-comparativo, transversal, ambispectivo de 2014 a 2015, para el cual se revisaron todos los expedientes médicos de pacientes con diagnóstico confirmado de EPOC por espirometría (realizada durante una campaña externa).

El centro de salud que incluyó a los pacientes cuenta con los servicios de atención clínica de consulta general, psicología, odontología, laboratorio, farmacia, imagenología (rayos X), trabajo social, inmunizaciones y enfermería, dirigida a una jurisdicción sanitaria; también ofrece formación de recursos humanos para médicos de pregrado y especialistas. Consta de 10 consultorios, un aula de enseñanza, un auditorio, biblioteca, espacios administrativos y de archivo clínico. Durante el 2015 brindó un promedio de 37,563 consultas anuales.

Para el proceso de atención, los pacientes se presentan a consulta de primera vez a través de la solicitud de una cita, se abre expediente y se elabora la historia clínica. En algunos pacientes, cuando el caso lo amerita, (por infraestructura, estudios complementarios, interconsultas, necesidad de internamiento, gravedad) es referido a un hospital de segundo o de tercer nivel.

En el caso de la atención de pacientes respiratorios crónicos como EPOC se dispone de espirómetro; empero, no existe personal encargado en su manejo y resguardo, por lo que el uso es limitado. Esto es parte de los motivos por los que no existen registros sistemáticos y confiables de EPOC; además, dicho diagnóstico se engloba en la categoría de «enfermedades respiratorias crónicas» que abarcan asma, fibrosis, tuberculosis, entre otras. Para EPOC hay una estrategia de referencia y contrarreferencia para continuar el control y seguimiento. El cálculo de una estimación de pacientes con EPOC partiendo de la muestra de pacientes atendidos tendría que corresponder a lo que se comenta en la literatura, un 7.8 a $20.6 \%{ }^{4,6} \mathrm{Al}$ presente, las condiciones actuales del centro de salud obligan a que la única forma de acercamiento a esta población sea mediante la revisión de las hojas de atención diaria de consulta, que es el único documento oficial en el que se establece el diagnóstico de EPOC.

Los criterios de inclusión fueron: diagnóstico confirmado de EPOC por espirometría, síntomas y factores de riesgo, expedientes con reporte de EPOC en la hoja de atención diaria, sin importar otros diagnósticos adicionales o comorbilidades. El criterio de exclusión fue por medio de expedientes con sólo sospecha de EPOC por falta de espirometría; y como criterio de eliminación expedientes con notas incompletas o ilegibles.

Como criterio externo de las recomendaciones se utilizó la Guía de Diagnóstico y Tratamiento de la Enfermedad Pulmonar Obstructiva Crónica del Gobierno Federal, del Catálogo Maestro de Guías de Práctica Clínica (Guías del CENETEC), la cual fue realizada, revisada y validada por neumólogos nacionales expertos y está basada en las Guías Globales GOLD. ${ }^{14}$ Cabe resaltar que todos los médicos que integran la clínica de atención primaria conocen dicha guía.

La recolección de datos se vertió en una ficha de identificación con variables sociodemográficas (edad, sexo, peso, talla) y del médico tratante (edad, sexo, grado académico y turno). También se diseñó una «hoja de cotejo" en la que se enumeraron las recomendaciones de primer nivel de atención de la GPC para EPOC, y se evaluaron de manera dicotómica (sí o no) con base en su cumplimiento por parte del médico tratante.

Se consideraron como variables 46 recomendaciones contenidas en la GPC agrupadas en siete áreas: a) factores de riesgo, 11 ; b) diagnóstico clínico, 6; c) estudios complementarios, 5; d) prevención, 8; e) tratamiento, 10 ; f) criterios de referencia, 5; y g) seguimiento, 1. Se calculó el porcentaje de cumplimiento por área de recomendaciones con una regla de tres, considerando como $100 \%$ el número total de recomendaciones por área evaluada, y como variable el número de recomendaciones llevadas a cabo por el médico tratante. Los pacientes que fueron valorados por más de un médico tratante se capturaron como un solo expediente; pero cada nota se evaluó de manera independiente en función del momento de atención. Para ejemplificar el proceso de cotejo tenemos: si el paciente se encontraba en fase de diagnóstico se consideraban las recomendaciones concernientes a factores de riesgo, diagnóstico clínico, estudios complementarios y prevención.

Este protocolo no fue sometido a Comité de Investigación debido a que no tiene implicación de intervenciones de ningún tipo, por ausencia de riesgos, no interfiere en la atención médica y por la protección de la confidencialidad de los sujetos.

\section{Análisis estadístico}

Se obtuvieron las frecuencias y porcentajes de cumplimiento para cada recomendación de la GPC, y por subgrupos de recomendaciones con base en el área 
de pertenencia (prevención, diagnóstico, tratamiento y referencia); y se realizaron análisis de comparación no paramétricos entre tres grupos independientes (médicos generales, médicos familiares y residentes) a través de Kruskal Wallis.

\section{RESULTADOS}

Durante el año 2015 fueron atendidos 6,693 pacientes mayores de 40 años, de ellos se estimarían alrededor

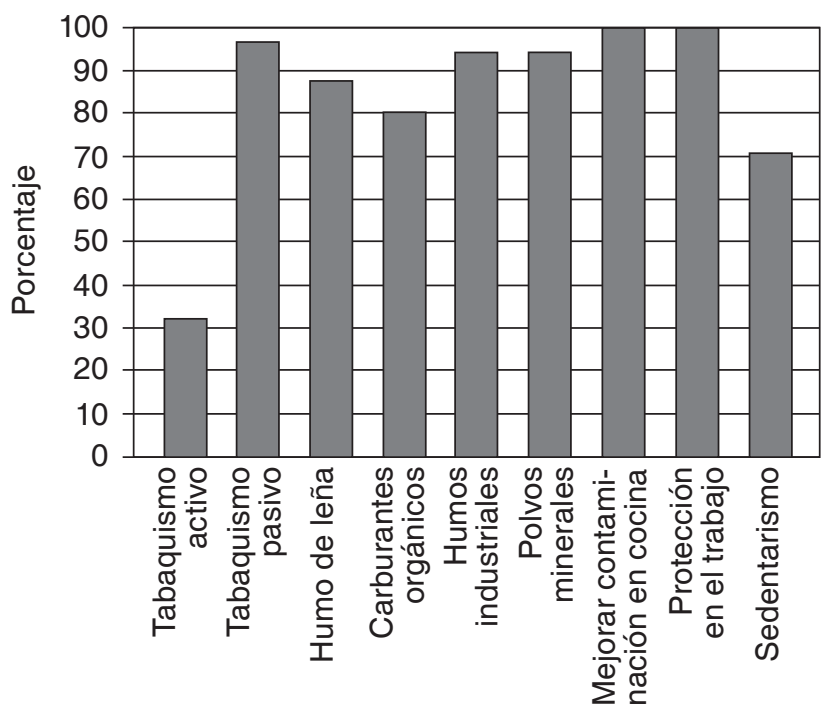

Recomendaciones de Guía de Práctica Clínica

Figura 1. Porcentajes de no cumplimiento de las recomendaciones de GPC-EPOC en factores de riesgo.

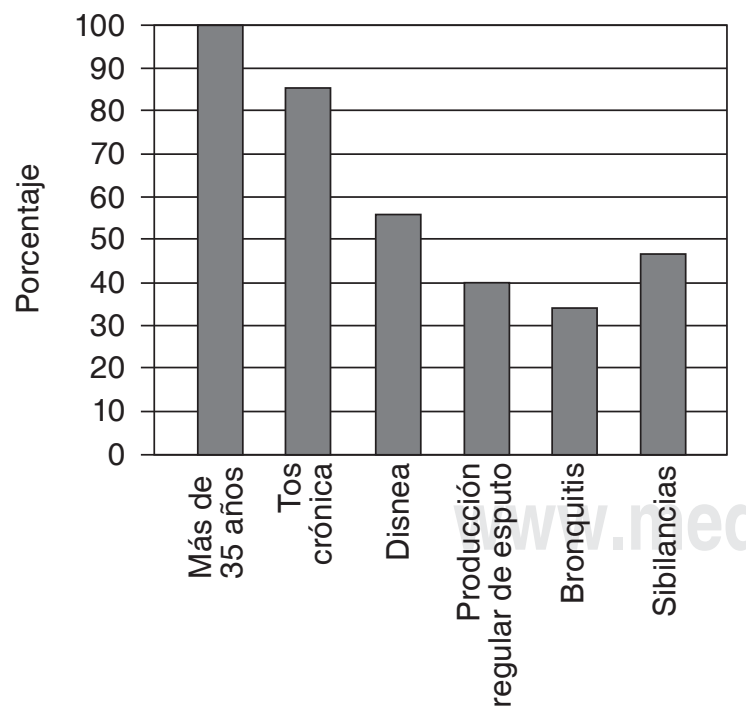

Recomendaciones de la Guía de Práctica Clínica

Figura 2. Porcentaje de no cumplimiento de criterios diagnósticos de la GPC-EPOC. de 522 casos de EPOC; sin embargo, el número de pacientes identificados fue de menos del $10 \%$ de ese estimado.

Se identificaron e incluyeron 25 expedientes de pacientes con diagnóstico de EPOC confirmado por espirometría; de éstos 5 (20\%) fueron mujeres y 20 $(80 \%)$ varones. El promedio de edad fue $66.5 \pm 14$ años y el índice de masa corporal (IMC) de $26 \pm 5$ $\mathrm{kg} / \mathrm{m}^{2}$.

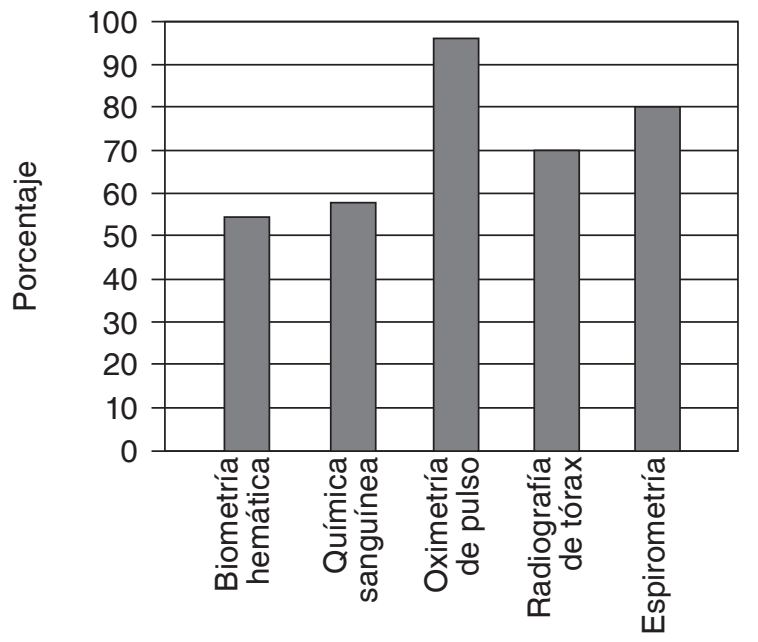

Recomendación de la Guía de Práctica Clínica

Figura 3. Porcentaje de no cumplimiento de las recomendaciones de la GPC-EPOC para estudios indicados al diagnóstico.

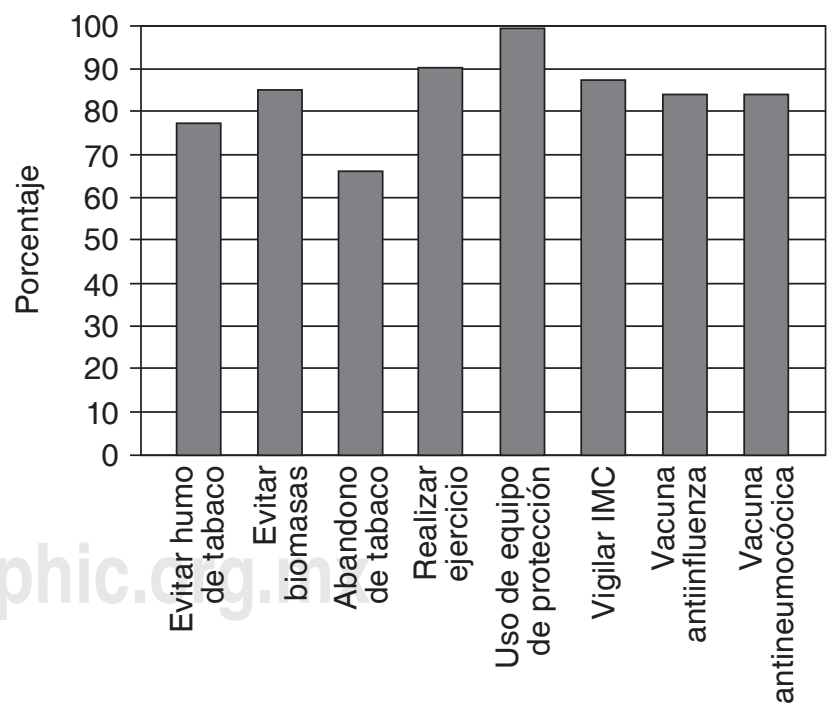

Recomendaciones de Guía de Práctica Clínica

Figura 4. Porcentajes de no cumplimiento de medidas de acción preventivas en personas con EPOC de acuerdo con la GPC. 
De los 25 expedientes se obtuvieron 36 notas médicas, el $67.6 \%$ de las notas fueron elaboradas por médicos mujeres. En cuanto a la formación de los médicos, 9 (35\%) eran médicos generales adscritos; 6 (16.7\%), 8 $(22.2 \%)$ y $1(2.8 \%)$ fueron médicos residentes de primer, segundo y tercer año de medicina familiar, respectivamente; y 12 (33.3\%) médicos familiares adscritos.

Las figuras 1 a 7 incluyen los porcentajes de cumplimiento de las recomendaciones. En la figura 5 se reportan las recomendaciones seguidas para el tratamiento. Debido al gran número de recomendaciones de este apartado, se enumeraron de acuerdo al siguiente código $0^{\mathbb{P}}$ : 1: indicación de broncodilatadores de corta acción como tratamiento de elección; 2: broncodilatadores de larga acción en pacientes con EPOC moderado o grave con exacerbaciones; 3: broncodilatadores de larga acción en pacientes que permanecen sintomáticos con el uso de broncodilatadores de corta acción; 4: broncodilatadores inhalados; 5: anticolinérgicos de corta duración agregados en pacientes que permanecen sintomáticos con el uso de broncodilatadores $\beta 2$ adrenérgicos; 6 : anticolinérgicos de larga duración; 7: glucocorticoides inhalados en pacientes con EPOC grave y más de dos recaídas; 8: glucocorticoides sistémicos; 9: antitusígeno; y 10: antibiótico en caso de infección o incremento de esputo.

De acuerdo con la formación del médico tratante hubo diferencias estadísticamente significativas entre los grupos en las áreas de factores de riesgo, prevención y criterios de referencia, todos ellos con mejor apego en el grupo de médicos residentes de medicina familiar (figura 7).

\section{DISCUSIÓN}

El objetivo del presente estudio fue determinar el cumplimiento de las GPC de EPOC por el personal médico

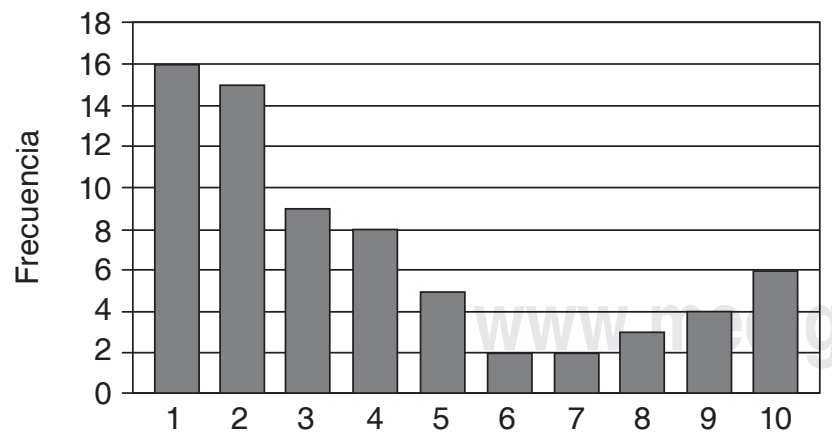

『Ver criterios en el texto.

Recomendaciones de la Guía de Práctica Clínica

Figura 5. Frecuencia de no cumplimiento de las medidas de tratamiento en personas con EPOC de acuerdo con la GPC. de un centro de salud. En estudios anteriores sobre la adherencia a las Guías de EPOC, se ha demostrado un bajo apego a pesar de que su diseño se basa en encuestas de autorreporte por parte de los médicos. Una de las principales fortalezas de este estudio es que

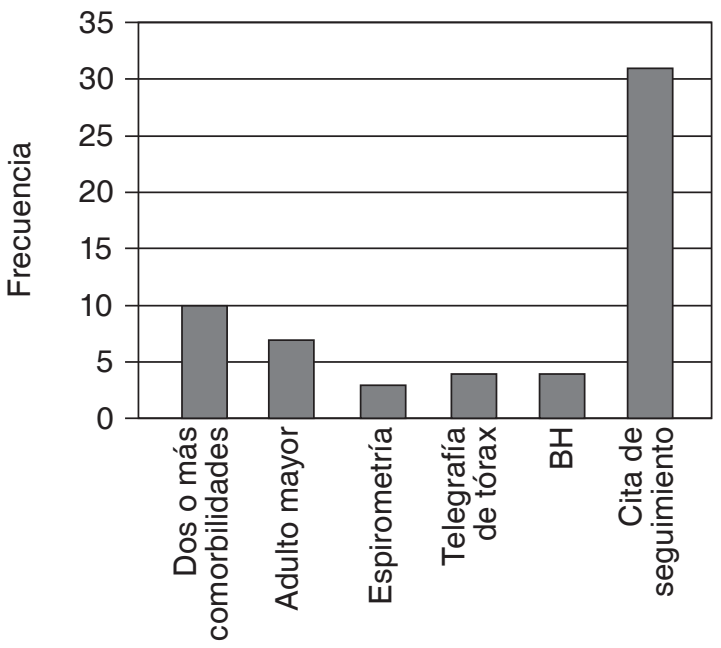

Recomendaciones de Guía de Práctica Clínica

Figura 6. Frecuencia de no cumplimiento de las recomendaciones de referencia y seguimiento en personas con EPOC de acuerdo con la GPC.

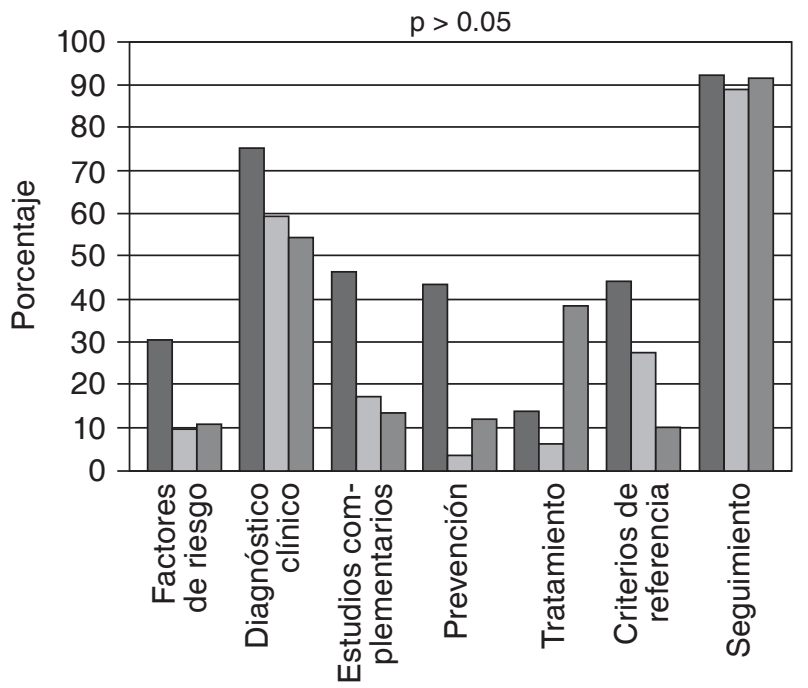

Apartados de recomendaciones de la GPC
$\square$ Residentes medicina familiar
$\square$ Médicos generales adscritos
$\square$ Médicos familiares adscritos

Fuente: Lista de cotejo.

Figura 7. Comparación de cumplimiento de la GPC según el perfil del médico tratante. 
está basado en la revisión de expedientes clínicos de pacientes atendidos en primer nivel. De acuerdo con las Guías de la OMS y la GPC, la atención de primer contacto es la columna vertebral del sistema de salud, por lo que implica la prevención, detección y atención de las enfermedades crónicas como la EPOC; además de la importancia de la creación de ambientes más saludables y la sensibilización acerca de estas enfermedades en ese primer nivel. ${ }^{1,14}$

De acuerdo con el grado académico de cada médico que labora en el centro de salud, los médicos residentes cumplieron con el total de las variables estudiadas de la GPC de EPOC en un $49.6 \%$. Los médicos familiares adscritos cumplieron en un $33.2 \%$, mientras que los médicos generales adscritos cumplieron con un $31.6 \%$. Estos resultados son similares a los reportados en otros estudios, en donde se ha encontrado que a pesar de la vasta información con que se cuenta hoy en día, los médicos refieren tener un conocimiento limitado de las guías y de su manejo. Situación que repercute sobre la calidad de atención brindada a los pacientes con EPOC (estimada muy por debajo a lo recomendado en las guías). ${ }^{15}$ Las principales razones de la falta de adherencia que argumentan los médicos están el hecho de que nunca las han leído, dificultades para obtenerlas o la falta de tiempo para revisarlas. ${ }^{13}$

El desconocimiento y pobre apego a las guías se reflejó en algunos resultados arrojados en el estudio PLATINO, donde se evaluó la prevalencia de EPOC en cinco ciudades de América Latina, entre ellas la Ciudad de México. Se reportó que el $24.7 \%$ de los médicos emite el consejo antitabáquico, el $13.35 \%$ ofrece un tratamiento farmacológico inhalado y el $43 \%$ llevó a cabo una evaluación respiratoria correcta; ${ }^{3}$ lo que habla de un subdiagnóstico y un subtratamiento de la EPOC.

La identificación de la exposición directa o indirecta a factores de riesgo reportada en estudios internacionales ${ }^{16,17}$ y en este estudio resulta alarmante; hace evidente una falta de atención en la indicación de medidas de prevención secundaria, así como poca sistematización en el proceso de valoración y diagnóstico que impiden tener una línea basal adecuada. Limita el compromiso de los pacientes con el control de su enfermedad pues no se establece una relación clara entre su conducta y la enfermedad, además, obstaculiza la implementación exitosa de medidas necesarias para disminuir o detener la exposición a los factores de riesgo que favorecen el desarrollo de la enfermedad. ${ }^{18}$

Por otro lado, la falta de registro de los síntomas como la disnea, la producción de esputo o las sibilancias que se observó en las notas del diagnóstico de los pacientes, dificulta la sospecha de la presencia de EPOC; y representa un problema para evaluar la evolución del paciente al no contar con indicadores objetivos de su estado de salud desde la valoración inicial del paciente. Es decir, resulta imposible conocer con certeza el efecto del manejo establecido y los requerimientos del paciente durante el seguimiento. ${ }^{19}$

Sumado a lo anterior, la clara evidencia de la falta de solicitud de estudios complementarios se relaciona y ocasiona directamente con la alta frecuencia de subdiagnóstico o diagnóstico erróneo; situación similar a lo reportado sobre la baja aptitud de médicos en la atención clínica ${ }^{19}$ al dejar a un lado una medición basal que permita valorar la evolución del paciente y favorecer el control de su sintomatología.

En el estudio IBERPOC realizado en España se encontró que sólo un $19.3 \%$ de todos los pacientes con diagnóstico de EPOC estable está tratado de manera adecuada; dato replicado a lo reportado en este estudio donde ninguna de las recomendaciones de tratamiento excede el $20 \%$ de su cumplimiento, lo que seguramente impide el control de la enfermedad. En el IBERPOC se halló, además, que sólo la mitad (49.3\%) de los pacientes con mayor gravedad están tratados adecuadamente. El mal manejo inicial y la mala evolución de los pacientes encarece los costos del sistema de salud y condiciona mayor impacto en su calidad de vida, mientras que un tratamiento adecuado desde el inicio favorecería la mejoría del paciente y la reducción de riesgo de complicaciones. ${ }^{20}$

Estos hallazgos difieren de manera importante con el objetivo del primer nivel de atención. Al ser poco sistematizada dista de tener la calidad técnica buscada con las guías y dificulta la satisfacción de las necesidades del paciente.

Estos resultados son preocupantes si consideramos la expectativa de vida que se ha descrito en los pacientes con EPOC, quienes tienen una menor esperanza de vida en comparación con los sujetos de la misma edad que no padecen la enfermedad. En este sentido se ha determinado que el $26 \%$ de los pacientes con EPOC fallece dentro de los siguientes ocho años a partir del diagnóstico de EPOC; además, entre más temprano se adquiere la enfermedad, mayor es el número de años de esperanza de vida perdidos, existiendo el riesgo de tener hasta un $60 \%$ de esperanza de vida perdida si se tiene EPOC a los 45 años. ${ }^{8}$

A la luz del impacto de la enfermedad, no hay duda de la necesidad de impulsar la capacitación continua del personal médico a través de la implementación de programas. Lo detectado en este centro de salud puede estar ocurriendo en muchos más, siendo ésta una muy buena oportunidad de corregir y equipar estos sitios. 


\section{Limitaciones del estudio}

Dentro de las limitaciones de nuestro estudio podemos comentar el número reducido de expedientes revisados. De acuerdo con el cálculo del tamaño de la muestra, el número de expedientes revisados fue muy por debajo de la estimación de pacientes con EPOC, lo que limita las interpretaciones y generalizaciones que se puedan hacer sobre los resultados; sin embargo, este hecho incluso podría ser un reflejo de un subdiagnóstico de la enfermedad en el propio centro. De cualquier forma representa un gran esfuerzo conocer la conducta de los médicos tratantes en su práctica diaria evitando posibles sesgos, como el saber que están siendo evaluados, tal como resulta en una entrevista o encuesta, y que puede interferir con las respuestas de los médicos.

Una observación interesante fue que los médicos residentes de medicina familiar tuvieron un significativo mayor apego a la GPC con respecto a los otros médicos evaluados, quizá debido a su condición de estudiantes que siguen desempeñando y que los coloca, casi siempre, en un proceso de evaluación que les exige reportar con más detalle sus acciones; no así los médicos adscritos que dejan pasar por alto este registro por la pérdida de interés. Debería considerarse en futuros estudios evaluar el cumplimiento de las GPC a través de una lista de cotejo similar a la realizada; pero cuya valoración sea al momento en que se está llevando a cabo la consulta para tener mayor certeza de la postura de los médicos y poder integrar mejor la información que reportan en la nota y su conducta durante la exploración física.

\section{CONCLUSIÓN}

Con base en los resultados arrojados en este estudio es claro el bajo apego a la GPC para EPOC por parte de los médicos de primer nivel de atención. Destacamos la importancia de implementar programas como AIRE (Acción Integrada por la Respiración para el Diagnóstico y Tratamiento de las Enfermedades Respiratorias) tomando como modelo el programa PAL. Así, el primer nivel de atención se vería obligado a realizar una adecuada historia clínica, hacer énfasis en las medidas de prevención, diagnóstico y tratamiento, además, dominar los criterios de referencia y continuidad del manejo de los pacientes. De lo contrario perpetúan el impacto de la EPOC en la salud pública debido al subdiagnóstico y subtratamiento; ${ }^{21}$ a través de acciones concretas como evitar el uso de sintomáticos respiratorios de poca utilidad y la subutilización del espirómetro, fomentar el consejo antitabaco y de medicamentos contra el tabaquismo, ${ }^{22}$ adoptando estrategias simples que han funcionado en otras patologías pulmonares como el caso de la tuberculosis.

\section{Conflicto de intereses}

La autora principal de este artículo original y otro de los coautores (responsable directo del área de Medicina Familiar) laboran en el centro de salud donde se llevó a cabo el estudio, pero no están incluidos dentro de los médicos evaluados. Los dos autores restantes, no laboran en el centro de salud. No existe conflicto de intereses para ninguno de los autores.

\section{REFERENCIAS}

1. World Health Organization. Enfermedad pulmonar obstructiva crónica (EPOC). [Internet]. Ginebra: 2012 [cited 2015 Jun 7]. Report No.: Nota descriptiva N .315. Available from: URL: http://www.who.int/mediacentre/ factsheets/fs315/es/index.html

2. National Heart, Lung, and Blood Institute. ¿Qué es la enfermedad pulmonar obstructiva crónica EPOC? [Internet]. USA: NHLBI; 2014 [cited 2015 Jul 12]. Report No.: Explore EPOC. Available from: URL: http://www.nhlbi. nih.gov/health-spanish/health-topics/temas/copd

3. Menezes AM, Perez-Padilla R, Jardim JR, et al.; PLATINO Team. Chronic obstructive pulmonary disease in five Latin American cities (the PLATINO study): a prevalence study. Lancet 2005;366(9500):1875-1881.

4. Tálamo C, de Oca MM, Halbert R, et al.; PLATINO Team. Diagnostic labeling of COPD in five Latin American cities. Chest 2007;131(1):60-67.

5. Miravitlles M, Soler-Cataluña JJ, et al. Guía española de la EPOC (GesEPOC). Actualización 2014. Arch Bronconeumol 2014;50 Suppl 1:1-16. doi:10.1016/S03002896(14)70070-5.

6. Salas HJ. Informe anual de autoevaluación del Director General del Instituto Nacional de Enfermedades Respiratorias Ismael Cosío Villegas [Internet]. Ciudad de México. Report No.: 2014. Fecha de consulta: 27 de julio, 2015. Accesible en: http://www.iner.salud.gob. mx/media/71140/2013.pdf

7. Vos T, Flaxman A, Naghavi M, et al. Years lived with disability (YLDS) for 1160 sequelae of 289 diseases and injuries 1990-2010: a systematic analysis for the Global Burden of Disease Study 2010. Lancet 2012;380(9859):21632196. doi: 10.1016/S0140-6736(12)61729-2.

8. Cuarto Consenso Mexicano para el Diagnóstico y Tratamiento de la EPOC. Guías para el Diagnóstico y Tratamiento de la Enfermedad Pulmonar Obstructiva Crónica. Neumol Cir Torax 2012;71(Supl 1):8-89.

9. Miravitlles M, Murio C, Guerrero T, Gisbert R; DAFNE Study Group. Decisiones sobre Antibioticoterapia y Farmacoeconomía en la EPOC. Pharmacoeconomic evaluation of acute exacerbations of chronic bronchitis and COPD. Chest 2002;121(5):1449-1455. 
10. Reynales-Shigematsu LM, Juárez-Márquez SA, ValdésSalgado R. Costos de atención médica atribuibles al tabaquismo en el IMSS, Morelos. Salud Publica Mex 2005;47(6):451-457.

11. Damiani M, Dixon J. Managing the pressure. Emergency hospital admissions in London 1991-2001 [Internet]. London, England: Kings Fund; 2012 [cited 2015 Sep 29]. Available from: http://www.kingsfund.org.uk/

12. Pérez-Padilla R, Stelmach R, Soto-Quiroz M, Cruz AA. Fighting respiratory diseases: divided efforts lead to weakness. J Bras Pneumol 2014;40(3):207-210.

13. Laniando-Laborín $R$, Rendón A, Alcantar-Schramm $\mathrm{J}$, Cazares-Adame R, Bauerle O. Subutilization of COPD guidelines in primary care: a pilot study. J Prim Care Community Health 2013;4(3):172-176. doi: $10.1177 / 2150131913475817$.

14. Consejo de Salubridad General, IMSS, ISSSTE, et al. Guía de Referencia Rápida. Diagnóstico y tratamiento de la enfermedad pulmonar obstructiva crónica [Internet]. México; 2011. (Catálogo Maestro de Guías de Práctica Clínica: IMSS-037-08). Fecha de consulta: 28 de julio, 2015. Accesible en: http://www.cenetec.salud.gob. mx/descargas/gpc/CatalogoMaestro/037_GPC_EPOC/ IMSS_037_08_GRR.pdf

15. Aisanov Z, Bai C, Bauerle O, et al. Primary care physician perceptions on the diagnosis and management of chronic obstructive pulmonary disease in diverse regions of the world. Int J Chron Obstruct Pulmon Dis 2012;7:271-282. doi: 10.2147/COPD.S28059.

16. Organización Mundial de la Salud. Prevención y control de las enfermedades no transmisibles: Directrices para la atención primaria en entornos con pocos recursos [Internet]. Ginebra: 2013. 68 p. Fecha de consulta: 10 de enero, 2015. Accesible en: http://apps.who.int/iris/ bitstream/10665/112396/1/9789243548395_spa.pdf

17. National Institute on Drug Abuse. ¿Cuáles son las consecuencias médicas del uso del tabaco? [Internet].
USA: National Institute on Drug Abuse; 2010 [cited 2015 Mar 10] p. 12. (Adicción al Tabaco). Available from: http://www.drugabuse.gov/es/publicaciones/ serie-de-reportes/adiccion-al-tabaco/cuales-son-lasconsecuencias-medicas-del-uso-del-tabaco

18. Consejo Nacional de Población. Partida V. Proyecciones de la población de México 2005-2050 [Internet]. México: 2006. Fecha de consulta: 20 de octubre, 2015. Accesible en: http://www.conapo.gob.mx/work/models/CONAPO/ proyecciones_estatales/Proy05-50.pdf

19. Casas D, Rodríguez A, Tapia E, Camacho E, Rodríguez R. Aptitud clínica en médicos familiares apegadas a guías de práctica clínica. Rev Mex Investig 2014;2(2):100-106.

20. Sobradillo V, Miravitlles M, Jiménez CA, et al. Estudio IBERPOC en España: prevalencia de síntomas respiratorios habituales y de limitación crónica al flujo aéreo. Arch Bronconeumol 1999;35(4):159-166. doi: 10.1016/ S0300-2896(15)30272-6

21. Pérez-Padilla R. Hacia un programa de prevención y atención primaria de enfermedades respiratorias, AIRE (Acción Integrada por la Respiración). Neumol Cir Torax 2012;71(3):244-249.

22. Centro Nacional de Excelencia Tecnológica en Salud. Diagnóstico y tratamiento de enfermedad obstructiva [Internet]. México: Secretaría de Salud; 2009. Fecha de consulta: 10 de abril, 2015. Accesible en: www.cenetec. salud.gob.mx

\author{
$\triangle$ Correspondencia: \\ Dra. Eloisa González \\ Rancho Piomo Núm. 18, interior 401, \\ colonia Nueva Oriental Coapa, 4300, \\ Delegación Tlalpan, Ciudad de México. \\ Correo electrónico: elimedic08@hotmail.com
}

Los autores declaran no tener conflicto de intereses. 